\title{
A Fuzzy Goal Programming Approach to Fully Fuzzy Linear Regression
}

\author{
Boris Pérez-Cañedo $^{1(\otimes)}\left(\mathbb{D}\right.$, Alejandro Rosete ${ }^{2}$ (D) José Luis Verdegay $^{3}$ (D), \\ and Eduardo René Concepción-Morales ${ }^{1}$ (D) \\ 1 University of Cienfuegos, Cienfuegos 55100, Cuba \\ bpcanedo@gmail.com, econcepm@gmail.com \\ 2 Technological University of Havana, Havana, Cuba \\ rosete@ceis.cujae.edu.cu \\ 3 University of Granada, Granada, Spain \\ verdegay@ugr.es
}

\begin{abstract}
Traditional linear regression analysis aims at finding a linear functional relationship between predictor and response variables based on available data of a given system, and, when this relationship is found, it is used to predict the future behaviour of the system. The difference between the observed and predicted data is supposed to be due to measurement errors. In fuzzy linear regression, on the other hand, this difference is supposed to be mainly due to the indefiniteness of the system. In this paper, we assume that predictor and response variables are LR-type fuzzy numbers, and so are all regression coefficients; this is known as fully fuzzy linear regression (FFLR) problem. We transform the FFLR problem into a fully fuzzy multiobjective linear programming (FFMOLP) problem. Two fuzzy goal programming methods based on linear and Chebyshev scalarisations are proposed to solve the FFMOLP problem. The proposed methods are compared with a recently published method and show promising results.
\end{abstract}

Keywords: Fully fuzzy linear regression - Fully fuzzy multiobjective linear programming $\cdot$ Fuzzy goal programming $\cdot$ Linear scalarisation • Chebyshev scalarisation

\section{Introduction and Preliminaries}

Traditional linear regression is one of the most frequently applied technique for finding functional relationships between predictor and response variables, and for making predictions. However, decision problems arising in ever-changing environments are difficult to describe or formulate with precise terms. Expert knowledge then gains a special value, and the need for its introduction into classical decision-making techniques has motivated the appearance and development of several mathematical theories dealing with uncertainty and vagueness. Among those theories, Fuzzy Sets Theory [31] has succeeded in numerous practical situations and is now an established research field. 
Fuzzy linear regression is a natural extension of the classical regression analysis and allows to predict the future behaviour of systems whose structure is not well defined and/or is influenced by subjectivity. It is particularly useful to forecast, e.g., future demands, resource availability and prices that could then be used to set up fuzzy optimisation problems in areas such as production, transportation, project management and so forth. Fuzzy linear regression has been used to forecast airport demand [21], oil consumption [1], house prices [32], sales [5] and short-term load in power distribution systems [24]. Several other applications are reported in [6].

Numerous fuzzy linear regression models and methods have been developed since the 1980s. Tanaka et al. [25] introduced fuzzy linear regression analysis and formulated a regression problem with crisp predictor variables, fuzzy response variable and fuzzy coefficients as a conventional linear programming problem. A modified version of Tanaka et al.'s [25] fuzzy linear regression method allowing negative spreads in the parameters was proposed in [3]. Chang and Lee [2] proposed fuzzy least square deviation and least absolute deviation models based on ranking functions. A multiobjective approach was proposed by Sakawa and Yano [23] by simultaneous consideration of the model fit and fuzziness. Recent methods for fuzzy linear regression have been presented in [14,18,22]. A comprehensive review until year 2019 is provided by Chukhrova and Johannssen [6].

So far, fuzzy linear regression methods mainly resort to the minimisation of crisp-valued distance functions between fuzzy numbers, either by direct generalisations of known crisp distance functions or by the use of linear ranking functions to defuzzify response observations and model predicted values, and then taking the absolute value of the difference as the distance between the two fuzzy numbers. A simulation study, considering distance functions from both approaches, was conducted in [14] to determine the best distance function in fuzzy linear regression using Monte Carlo methods. Notably, Voxman [26] has argued that the distance between two fuzzy numbers should also be a fuzzy number, and proposed a fuzzy-valued distance function. However, to the best of our knowledge, fuzzy-valued distance functions have not been used in fuzzy regression analysis.

In this paper, we seek to evaluate other models and methods for fuzzy linear regression analysis, which do not rely on crisp-valued distance functions. We propose two methods based on FFMOLP for fuzzy linear regression analysis, in which the predictor variables, response variable and regression coefficients are LR-type fuzzy numbers. The proposed methods rely on the lexicographic approach to fully fuzzy linear programming (FFLP) with inequality constraints recently proposed in [19]. The rest of the paper is organised as follows. Section 1.1 presents some fundamental definitions on LR-type fuzzy numbers. Section 1.2 outlines the lexicographic method [19] for solving FFLP problems. In Sect.2, we formulate the FFLR problem as a FFMOLP problem, and propose two fuzzy scalarisation methods based on classical goal programming to solve it. Section 3 discusses a numerical example. Lastly, Sect. 4 presents the conclusions and remarks for future work. 


\subsection{LR-type Fuzzy Numbers}

Dubois and Prade [8] defined the concept of LR-type fuzzy number and proposed simple formulae for arithmetic operations. In this section, we present some definitions concerning LR-type fuzzy numbers taken from reference [8].

Definition 1. A fuzzy number $\tilde{a}=(m, \alpha, \beta)_{L R}$ is said to be an LR-type fuzzy number if its membership function is given by:

$$
\mu_{\tilde{a}}(x)=\left\{\begin{array}{l}
L\left(\frac{m-x}{\alpha}\right) \\
R\left(\frac{x-n}{\beta}\right) m \leq x \leq m+\beta, \beta>0
\end{array}\right.
$$

where $m$ is the modal value; $L$ and $R$ (called left and right reference functions, respectively) are non-increasing functions $[0,+\infty) \rightarrow[0,1]$, fulfilling $L(0)=R(0)=1 ; \alpha$ and $\beta$ are the left and right spreads of $\tilde{a}$, respectively. The set of all LR-type fuzzy numbers, defined on $\Re$, is denoted by $\mathcal{F}(\Re)$.

Definition 2. Let $\tilde{a}_{1}=\left(m_{1}, \alpha_{1}, \beta_{1}\right)_{L R}$ and $\tilde{a}_{2}=\left(m_{2}, \alpha_{2}, \beta_{2}\right)_{L R}$ be any LR-type fuzzy numbers, then $\tilde{a}_{1}=\tilde{a}_{2}$ if and only if $m_{1}=m_{2}, \alpha_{1}=\alpha_{2}$ and $\beta_{1}=\beta_{2}$.

Definition 3. An LR-type fuzzy number $\tilde{a}=(m, \alpha, \beta)_{L R}$ is said to be nonnegative (resp. non-positive) if $m-\alpha \geq 0$ (resp. $m+\beta \leq 0$ ). This is denoted by $\tilde{a} \geq 0$ (resp. $\tilde{a} \leq 0)$.

Definition 4. An LR-type fuzzy number $\tilde{a}=(m, \alpha, \beta)_{L R}$ is said to be unrestricted if $m$ is an arbitrary real number.

Definition 5. Let $\tilde{a}_{1}=\left(m_{1}, \alpha_{1}, \beta_{1}\right)_{L R}$ and $\tilde{a}_{2}=\left(m_{2}, \alpha_{2}, \beta_{2}\right)_{L R}$ be two LR-type fuzzy numbers, then fuzzy addition is given by $\tilde{a}_{1} \oplus \tilde{a}_{2}=\left(m_{1}+m_{2}, \alpha_{1}+\alpha_{2}\right.$, $\left.\beta_{1}+\beta_{2}\right)_{L R}$.

Definition 6. The product of two non-negative LR-type fuzzy numbers $\tilde{a}_{1}=$ $\left(m_{1}, \alpha_{1}, \beta_{1}\right)_{L R}$ and $\tilde{a}_{2}=\left(m_{2}, \alpha_{2}, \beta_{2}\right)_{L R}$ is given by $\tilde{a}_{1} \odot \tilde{a}_{2}=\left(m_{1} m_{2}, m_{1} \alpha_{2}+\right.$ $\left.\alpha_{1} m_{2}-\alpha_{1} \alpha_{2}, n_{1} \beta_{2}+\beta_{1} n_{2}+\beta_{1} \beta_{2}\right)_{L R}$.

The reader is referred to [15] for the definition of the product of unrestricted LR-type fuzzy numbers.

\subsection{FFLP Problem and Lexicographic Solution Method}

Due to the vast number of practical situations where fuzzy quantities must be compared, ranking fuzzy numbers is still recognised as a fundamental research problem in Fuzzy Sets Theory. Many ranking methodologies have been proposed in the literature $[29,30]$. However, several researchers have noticed that most existing ranking methodologies cannot yield a total order of fuzzy numbers in a strict sense. To resolve this issue, lexicographic ranking criteria have been proposed as an alternative $[11,27,28]$. 
The integration of lexicographic ranking criteria into FFLP methods started with $[12,13]$ and has been recently investigated in $[7,9,10,16,19,20]$. In particular, the use of lexicographic ranking criteria for handling fuzzy inequality constraints has been proposed in $[12,19,20]$. In this section, we present the lexicographic method [19] for solving FFLP problems with inequality constraints. This method constitutes the basis of the results presented in the following sections.

Firstly, we need to introduce an order relation on $\mathcal{F}(\Re)$. Let $\tilde{a}=(m, \alpha$, $\beta)_{L R}$ be an arbitrary LR-type fuzzy number, and suppose we have three linear functions of the parameters of $\tilde{a}, f_{k}(\tilde{a}):=w_{k 1} m+w_{k 2} \alpha+w_{k 3} \beta$ for $k=1,2,3$. If each $w_{k r}$ is chosen such that matrix $\left[w_{k r}\right]$ is non-singular, then $\tilde{a}_{1}=\tilde{a}_{2}$ if and only if $f_{k}\left(\tilde{a}_{1}\right)=f_{k}\left(\tilde{a}_{2}\right)$ for $k=1,2,3$.

Based on the above idea, we may consider the following criterion for ranking LR-type fuzzy numbers.

Definition 7. Let $\leq_{\text {lex }}$ denote the lexicographic order relation on $\Re^{3}$. For any $\tilde{a}_{1}, \tilde{a}_{2} \in \mathcal{F}(\Re)$, the strict inequality $\tilde{a}_{1} \prec \tilde{a}_{2}$ holds, if and only if $\left(f_{k}\left(\tilde{a}_{1}\right)\right)_{k=1,2,3}<_{\text {lex }}\left(f_{k}\left(\tilde{a}_{2}\right)\right)_{k=1,2,3}$. The weak inequality $\tilde{a}_{1} \preceq \tilde{a}_{2}$ holds, if and only if $\left(f_{k}\left(\tilde{a}_{1}\right)\right)_{k=1,2,3}<_{\text {lex }}\left(f_{k}\left(\tilde{a}_{2}\right)\right)_{k=1,2,3}$ or $\left(f_{k}\left(\tilde{a}_{1}\right)\right)_{k=1,2,3}=\left(f_{k}\left(\tilde{a}_{2}\right)\right)_{k=1,2,3}$.

It can be shown that $\preceq$ satisfies the total order properties. That is, for all $\tilde{a}, \tilde{b}$ and $\tilde{c}$ in $\mathcal{F}(\Re)$ :

- $\tilde{a} \preceq \tilde{a}$ (reflexivity);

- $\tilde{a} \preceq \tilde{b}$ or $\tilde{b} \preceq \tilde{a}$ (comparability);

- if $\tilde{a} \preceq \tilde{b}$ and $\tilde{b} \preceq \tilde{c}$, then $\tilde{a} \preceq \tilde{c}$ (transitivity);

- if $\tilde{a} \preceq \tilde{b}$ and $\tilde{b} \preceq \tilde{a}$, then $\tilde{a}=\tilde{b}$ (anti-symmetry).

Next, we present the lexicographic method proposed in [19] for solving FFLP problems with equality and inequality constraints.

The FFLP problem can be formulated as follows, where $\tilde{c}_{j}, \tilde{a}_{i j}$ and $\tilde{b}_{i}$ are LR-type fuzzy parameters, $\tilde{x}_{j}$ denote the LR-type fuzzy decision variables, and $\preceq$ is an order relation on $\mathcal{F}(\Re)$; here, we assume that $\preceq$ is given by Definition 7 .

$$
\begin{aligned}
& \min \sum_{j=1}^{n} \tilde{c}_{j} \odot \tilde{x}_{j} \\
& \text { s.t. } \sum_{j=1}^{n} \tilde{a}_{i j} \odot \tilde{x}_{j}\{\preceq,=, \succeq\} \tilde{b}_{i} ; i=1,2, \ldots, m \\
& \quad \tilde{x}_{j} \in \mathcal{F}(\Re) ; j=1,2, \ldots, n
\end{aligned}
$$

By using Definitions 2 and 7, FFLP problem (1) is transformed into problem (2), which is then transformed into problem (3). To carry out these transformations, we have assumed that $\tilde{z}=\sum_{j=1}^{n} \tilde{c}_{j} \odot \tilde{x}_{j}, \tilde{a}_{i}=\left(m_{i}^{a}, \alpha_{i}^{a}, \beta_{i}^{a}\right)_{L R}=\sum_{j=1}^{n} \tilde{a}_{i j} \odot \tilde{x}_{j}$, $\tilde{b}_{i}=\left(m_{i}^{b}, \alpha_{i}^{b}, \beta_{i}^{b}\right)_{L R}$ and $\tilde{x}_{j}=\left(m_{j}^{x}, \alpha_{j}^{x}, \beta_{j}^{x}\right)_{L R}$. In addition, $I_{\mathrm{e}}, I_{\mathrm{le}}$ and $I_{\mathrm{ge}}$ denote 
the index sets of the fuzzy equality, less-than-or-equal-to and greater-than-orequal-to constraints of FFLP problem (1), respectively; $\epsilon$ and $M$ are positive real numbers sufficiently small and large, respectively.

$$
\begin{aligned}
\operatorname{lexmin} & \left(f_{k}(\tilde{z})\right)_{k=1,2,3} \\
\text { s.t. } & \left(f_{k}\left(\tilde{a}_{i}\right)\right)_{k=1,2,3}\left\{\leq_{l e x}, \geq_{l e x}\right\}\left(f_{k}\left(\tilde{b}_{i}\right)\right)_{k=1,2,3} ; i \in I_{\text {le }} \cup I_{\text {ge }} \\
& m_{i}^{a}=m_{i}^{b}, \alpha_{i}^{a}=\alpha_{i}^{b}, \beta_{i}^{a}=\beta_{i}^{b} ; i \in I_{\mathrm{e}} \\
& \alpha_{j}^{x} \geq 0, \beta_{j}^{x} \geq 0 ; j=1,2, \ldots, n
\end{aligned}
$$

$$
\begin{array}{ll}
\operatorname{lexmin} & \left(f_{k}(\tilde{z})\right)_{k=1,2,3} \\
\text { s.t. } & -M \sum_{p=1}^{k-1} y_{i p}+\epsilon y_{i k} \leq f_{k}\left(\tilde{b}_{i}\right)-f_{k}\left(\tilde{a}_{i}\right) \leq M y_{i k} ; i \in I_{\mathrm{le}}, k=1,2,3 \\
& -M \sum_{p=1}^{k-1} y_{i p}+\epsilon y_{i k} \leq f_{k}\left(\tilde{a}_{i}\right)-f_{k}\left(\tilde{b}_{i}\right) \leq M y_{i k} ; i \in I_{\mathrm{ge}}, k=1,2,3 \\
& m_{i}^{a}=m_{i}^{b}, \alpha_{i}^{a}=\alpha_{i}^{b}, \beta_{i}^{a}=\beta_{i}^{b} ; i \in I_{\mathrm{e}} \\
& y_{i k} \in\{0,1\} ; i \in I_{\mathrm{le}} \cup I_{\mathrm{ge}}, k=1,2,3 \\
& \alpha_{j}^{x} \geq 0, \beta_{j}^{x} \geq 0 ; j=1,2, \ldots, n
\end{array}
$$

Theorem 1. FFLP problem (1) is equivalent to problem (3).

Proof. See [19].

Remark 1. In order to solve FFLP problem (1), we must choose a lexicographic criterion for ranking LR-type fuzzy numbers. There are several such criteria in the literature (see, e.g., [11,27]). Notably, the solution method outlined here is general enough so as to allow a decision-maker to use the criterion that best fits the decision-making problem at hand.

\section{FFLR: Proposed Methods}

Let $\tilde{x}_{j}, \tilde{A}_{j}(j=0,2, \ldots, n)$ and $\tilde{y}$ be LR-type fuzzy numbers. Then the FFLR model is formulated as in Eq. (4).

$$
\tilde{y}=\tilde{A}_{0} \oplus \tilde{A}_{1} \odot \tilde{x}_{1} \oplus \tilde{A}_{2} \odot \tilde{x}_{2} \oplus \cdots \oplus \tilde{A}_{n} \odot \tilde{x}_{n}=\tilde{A}_{0} \oplus \sum_{j=1}^{n} \tilde{A}_{j} \odot \tilde{x}_{j}
$$

In Eq. (4), each $\tilde{x}_{j}$ is termed fuzzy predictor variable, $\tilde{y}$ fuzzy response variable and $\tilde{A}_{j}$ fuzzy regression coefficient. Now, let us consider a sample of LRtype fuzzy numbers $(\tilde{X} \mid \tilde{Y})$, where $\tilde{X}=\left(\tilde{x}_{i j}\right)_{\substack{i=1,2, \ldots, m \\ j=1,2, \ldots, n}}$ contains the observations corresponding to each fuzzy predictor variable $\tilde{x}_{j}$, and the column vector 
$\tilde{Y}=\left(\tilde{y}_{i}\right)_{i=1,2, \ldots, m}$ contains the observations of the fuzzy response variable $\tilde{y}$. We wish to determine the estimates of $\tilde{A}_{j}$ so as to obtain the best fitting model given the available data.

In what follows, we formulate the FFLR problem as a FFMOLP problem. To this aim, we introduce two non-negative fuzzy deviation variables $\tilde{S} p_{i}$ and $\tilde{S} n_{i}$ for each sample. Thus, the following set of fuzzy equalities is obtained.

$$
\tilde{A}_{0} \oplus \sum_{j=1}^{n} \tilde{A}_{j} \odot \tilde{x}_{i j} \oplus \tilde{S} p_{i}=\tilde{y}_{i} \oplus \tilde{S} n_{i} ; i=1,2, \ldots, m
$$

Therefore, we may consider the following FFMOLP problem:

$$
\begin{aligned}
\min & \left(\tilde{S} p_{1} \oplus \tilde{S} n_{1}, \tilde{S} p_{2} \oplus \tilde{S} n_{2}, \ldots, \tilde{S} p_{m} \oplus \tilde{S} n_{m}\right) \\
\text { s.t: } & \tilde{A}_{0} \oplus \sum_{j=1}^{n} \tilde{A}_{j} \odot \tilde{x}_{i j} \oplus \tilde{S} p_{i}=\tilde{y}_{i} \oplus \tilde{S} n_{i} ; i=1,2, \ldots, m \\
& \tilde{A}_{j} \text { unrestricted; } j=0,1, \ldots, n \\
& \tilde{S} p_{i} \geq 0, \tilde{S} n_{i} \geq 0 ; i=1,2, \ldots, m
\end{aligned}
$$

In order to solve (P1), we resort to two known classical scalarisation methods based on goal programming, which are extended to the fuzzy case: linear scalarisation method and Chebyshev (minimax) scalarisation method.

\subsection{Linear Scalarisation Method}

In this method, each objective function is multiplied by a positive weighting factor and the resulting expressions are added together. Thus, we have,

$$
\begin{aligned}
\min & \sum_{i=1}^{m} w_{i}\left(\tilde{S} p_{i} \oplus \tilde{S} n_{i}\right) \\
\text { s.t: } & \tilde{A}_{0} \oplus \sum_{j=1}^{n} \tilde{A}_{j} \odot \tilde{x}_{i j} \oplus \tilde{S} p_{i}=\tilde{y}_{i} \oplus \tilde{S} n_{i} ; i=1,2, \ldots, m \\
& \tilde{A}_{j} \text { unrestricted; } j=0,1, \ldots, n \\
& \tilde{S} p_{i} \geq 0, \tilde{S} n_{i} \geq 0 ; i=1,2, \ldots, m
\end{aligned}
$$

Hereafter, $w_{i}=1$ for $i=1,2, \ldots, m$ since no particular preference for the objective functions shall be considered.

\subsection{Chebyshev Scalarisation Method}

In this case the scalarising function is $\tilde{S}=\max \left(\tilde{S} p_{1} \oplus \tilde{S} n_{1}, \tilde{S} p_{2} \oplus \tilde{S} n_{2}, \ldots, \tilde{S} p_{m} \oplus\right.$ $\left.\tilde{S} n_{m}\right)$; hence, we have that $\tilde{S} p_{i} \oplus \tilde{S} n_{i} \preceq \tilde{S}$ for $i=1,2, \ldots, m$. By substituting 
into (P1), we obtain,

$$
\begin{array}{ll}
\min & \tilde{S} \\
\text { s.t: } & \tilde{A}_{0} \oplus \sum_{j=1}^{n} \tilde{A}_{j} \odot \tilde{x}_{i j} \oplus \tilde{S} p_{i}=\tilde{y}_{i} \oplus \tilde{S} n_{i} ; i=1,2, \ldots, m \\
& \tilde{S} p_{i} \oplus \tilde{S} n_{i} \preceq \tilde{S} ; i=1,2, \ldots, m \\
& \tilde{A}_{j} \text { unrestricted; } j=0,1, \ldots, n \\
& \tilde{S} \geq 0, \tilde{S} p_{i} \geq 0, \tilde{S} n_{i} \geq 0 ; i=1,2, \ldots, m
\end{array}
$$

thus, the objective is to minimise the maximal deviation.

\subsection{Steps of the Proposed FFLR Methods}

The whole procedure can be summarised in the following six steps.

1. Input: Sample data $\tilde{X}$ and $\tilde{Y}$;

2. choose a lexicographic criterion for ranking LR-type fuzzy numbers;

3. set up FFMOLP problem (P1);

4. choose either of the proposed scalarisation methods, and set up FFLP problem (l-P1) or FFLP problem (ch-P1);

5. solve the FFLP problem chosen in Step 4 by using the lexicographic method outlined in Sect. 1.2;

6. output: $\tilde{A}_{j}$ for $j=0,1, \ldots, n$ as the estimated regression parameters.

\section{Numerical Example}

The example in this section is taken from references $[4,17,18]$. The dataset contains 30 samples, each having four predictor variables and one response variable (see Table 1 ). It is a real-life dataset comprising triangular fuzzy numbers used to subjectively evaluate employee's performance according to work quality, inability to endure job stress, frequency of delays, and communication and coordination ability. As part of the solution procedure from Sect.2.3, the functions $f_{1}(\tilde{a}):=3 m+\beta-\alpha, f_{2}(\tilde{a}):=m+\beta$ and $f_{3}(\tilde{a}):=\alpha+\beta$ were used to define a lexicographic order relation on $\mathcal{F}(\Re)$.

We applied the proposed methods and obtained the following two models. The estimated responses of both models are shown in Table 2.

$$
\begin{aligned}
\tilde{y}_{\text {linear }}= & (0.85684,0,0)_{L R} \odot \tilde{x}_{1} \oplus(-0.12989,0,0)_{L R} \odot \tilde{x}_{2} \\
\oplus & (-0.17893,0.01697,0)_{L R} \odot \tilde{x}_{3} \oplus(0.04995,0,0)_{L R} \odot \tilde{x}_{4} \\
\oplus & (12.60258,0.14101,0)_{L R} \\
\tilde{y}_{\text {chebyshev }}= & (0.85662,0,0)_{L R} \odot \tilde{x}_{1} \oplus(-0.15074,0.03749,0)_{L R} \odot \tilde{x}_{2} \\
& \oplus(-0.14948,0,0)_{L R} \odot \tilde{x}_{3} \oplus(0.09081,0,0)_{L R} \odot \tilde{x}_{4} \\
& \oplus(10.03612,0,0)_{L R}
\end{aligned}
$$


Table 1. Sample dataset.

\begin{tabular}{|c|c|c|c|c|}
\hline$\tilde{x}_{1}$ & $\tilde{x}_{2}$ & $\tilde{x}_{3}$ & $\tilde{x}_{4}$ & $\tilde{y}$ \\
\hline$(50,8,8)_{L R}$ & $(98,6,2)_{L R}$ & $(71,9,11)_{L R}$ & $(70,11,13)_{L R}$ & $(30,11,9)_{L R}$ \\
\hline$L R$ & $(76,6,2)_{L R}$ & $(61,9,11)_{L R}$ & $(46,11,13)_{L R}$ & $(20,13,10)_{L R}$ \\
\hline$(41,8$ & $(88,6,2)_{L R}$ & $(73,9,11)_{L R}$ & $(58,11,13)_{L R}$ & $(25,11,12)_{L R}$ \\
\hline$(60,9$ & $2,9,10)_{L R}$ & $(79,9,6)_{L R}$ & $(66,8,9)_{L R}$ & $(45,12,10)_{L R}$ \\
\hline 7) $L R$ & $(50,9,10)_{L R}$ & $(75,9,6)_{L R}$ & $, 9)_{L R}$ & $(38,12,8)_{L R}$ \\
\hline$(59,9,7)_{L R}$ & $(60,9,10)_{L R}$ & $(85,9,6)_{L R}$ & $(64,8,9)_{L R}$ & $(43,11,9)_{L R}$ \\
\hline$(61,9,11)_{L R}$ & $(77,8,6)_{L R}$ & 8) $L R$ & $(18,7,13)_{L R}$ & $(40,17,11)_{L R}$ \\
\hline$(58,9,11)_{L R}$ & $(75,8,6)_{L R}$ & 3) $L R$ & $(16,7,13)_{L R}$ & $(38,11,12)_{L R}$ \\
\hline$(55,9,11)_{L R}$ & $(72,8,6)_{L R}$ & $(79,5,8)_{L R}$ & $(13,7,13)_{L R}$ & $(37,12,12)_{L R}$ \\
\hline$(66$, & $(59,17,11)_{L R}$ & $(39,8,9)_{L R}$ & $(83,14,11)_{L R}$ & $(60,11,12)_{L R}$ \\
\hline (69, & $(63,17,11)_{L R}$ & ) $L R$ & $4,11)_{L R}$ & $, 9)_{L R}$ \\
\hline$(59,8,7)_{L R}$ & $(53,17,11)_{L R}$ & $(39,8,9)_{L R}$ & $(77,14,11)_{L R}$ & $(54,11,8)_{L R}$ \\
\hline$(74,4,6)_{L R}$ & $(89,11,5)_{L R}$ & $(70,12,13)_{L R}$ & $(82,14,10)_{L R}$ & $(61,14,3)_{L R}$ \\
\hline$(41$, & 5) $L R$ & $(58,12,13)_{L R}$ & $(50,14,10)_{L R}$ & $(34,10,8)_{L R}$ \\
\hline$(49,2$ & & $(66,12,13)_{L R}$ & $(58,14,10)_{L R}$ & $(38,9,9)_{L R}$ \\
\hline$(76,8$ & & $(37,8,11)_{L R}$ & $(75,5,10)_{L R}$ & $(64,16,9)_{L R}$ \\
\hline$(57$, & & 11) $L R$ & $(56,5,10)_{L R}$ & $(56,13,7)_{L R}$ \\
\hline$(72$, & $, 10,8)_{L R}$ & $(33,8,11)_{L R}$ & $(71,5,10)_{L R}$ & $(63,11,9)_{L R}$ \\
\hline$(78,7,8)_{L R}$ & $(65,6,6)_{L R}$ & $(82,11,11)_{L R}$ & $(64,8,12)_{L R}$ & $(66,16,5)_{L R}$ \\
\hline$(58,7,8)_{L R}$ & & $(62,11,11)_{L R}$ & $(44,8,12)_{L R}$ & $(49,12,9)_{L R}$ \\
\hline$(72,7,8)_{L R}$ & $(59,6,6)_{L R}$ & $(76,11,11)_{L R}$ & $(58,8,12)_{L R}$ & $(55,10,12)_{L R}$ \\
\hline$(90,8,5)_{L R}$ & $(95,13,3)_{L R}$ & $(80,11,8)_{L R}$ & $(72,7,13)_{L R}$ & $(67,11,14)_{L R}$ \\
\hline$(68,8,5)_{L R}$ & $(73,13,3)_{L R}$ & $(58,11,8)_{L R}$ & $(50,7,13)_{L R}$ & $(53,10,9)_{L R}$ \\
\hline$(71,8,5)_{L R}$ & $(76,13,3)_{L R}$ & $(61,11,8)_{L R}$ & $(53,7,13)_{L R}$ & $(54,9,10)_{L R}$ \\
\hline$(92,8,6)_{L R}$ & $(76,6,9)_{L R}$ & $(78,10,6)_{L R}$ & $(27,9,15)_{L R}$ & $(70,13,7)_{L R}$ \\
\hline$(94,8,6)_{L R}$ & $(78,6,9)_{L R}$ & $(80,10,6)_{L R}$ & $(29,9,15)_{L R}$ & $(68,9,10)_{L R}$ \\
\hline$(87,8,6)_{L R}$ & $(71,6,9)_{L R}$ & $(73,10,6)_{L R}$ & $(22,9,15)_{L R}$ & $(65,10,9)_{L R}$ \\
\hline$(94,6,5)_{L R}$ & $(51,9,8)_{L R}$ & $(30,9,11)_{L R}$ & $(29,9,16)_{L R}$ & $(75,5,14)_{L R}$ \\
\hline$(95,6,5)_{L R}$ & $(52,9,8)_{L R}$ & $(31,9,11)_{L R}$ & $(30,9,16)_{L R}$ & $(84,10,7)_{L R}$ \\
\hline$(86,6,5)_{L R}$ & $(43,9,8)_{L R}$ & $(22,9,11)_{L R}$ & $(21,9,16)_{L R}$ & $(80,12,6)_{L R}$ \\
\hline
\end{tabular}

The obtained models' predicted values were compared with the ones reported by $\mathrm{Li}$ et al. [18], according to the overall absolute distance from the observed responses, using Eqs. (5) and (6).

$$
\begin{aligned}
d\left(\tilde{y}_{i}^{\text {obs }}, \tilde{y}_{i}^{\text {pred }}\right)= & \left|m_{i}^{\text {obs }}-m_{i}^{\text {pred }}\right|+\left|m_{i}^{\text {obs }}-\alpha_{i}^{\text {obs }}-\left(m_{i}^{\text {pred }}-\alpha_{i}^{\text {pred }}\right)\right| \\
& +\left|m_{i}^{\text {obs }}+\beta_{i}^{\text {obs }}-\left(m_{i}^{\text {pred }}+\beta_{i}^{\text {pred }}\right)\right|
\end{aligned}
$$


Table 2. Estimated values reported by $\mathrm{Li}$ et al. [18] and the ones obtained by using the proposed methods. The observed values have been kept to aid visual comparison.

\begin{tabular}{|c|c|c|c|}
\hline Observed response & Li et al. [18] & Chebyshev Scalarisation & Linear Scalarisation \\
\hline$(30,11,9)_{L R}$ & $(32.053,10.999,9.352)_{L R}$ & $(33.837,13.547,10.283)_{L R}$ & $(33.508,11.166,9.894)_{L R}$ \\
\hline$(20,13,10)_{L R}$ & $(17.915,10.916,9.352)_{L R}$ & $(18.480,12.722,10.283)_{L R}$ & $(18.962,10.996,9.894)_{L R}$ \\
\hline$(25,11,12)_{L R}$ & $(25.053,10.962,9.352)_{L R}$ & $(26.247,13.172,10.283)_{L R}$ & $(26.138,11.199,9.894)_{L R}$ \\
\hline$(45,12,10)_{L R}$ & $(45.501,12.046,8.795)_{L R}$ & $(46.271,13.540,9.516)_{L R}$ & $(45.121,12.068,9.227)_{L R}$ \\
\hline$(38,12,8)_{L R}$ & $(37.998,11.999,8.795)_{L R}$ & $(38.166,13.090,9.516)_{L R}$ & $(37.371,12.000,9.227)_{L R}$ \\
\hline$(43,11,9)_{L R}$ & $(43.947,12.038,8.795)_{L R}$ & $(44.638,13.465,9.516)_{L R}$ & $(43.350,12.170,9.227)_{L R}$ \\
\hline$(40,17,11)_{L R}$ & $(39.767,11.304,12.074)_{L R}$ & $(39.611,13.558,12.557)_{L R}$ & $(40.558,11.992,12.009)_{L R}$ \\
\hline$(38,11,12)_{L R}$ & $(37.874,11.296,12.074)_{L R}$ & $(37.609,13.483,12.557)_{L R}$ & $(38.684,11.941,12.009)_{L R}$ \\
\hline$(37,12,12)_{L R}$ & $(36.090,11.285,12.074)_{L R}$ & $(35.668,13.370,12.557)_{L R}$ & $(36.890,11.890,12.009)_{L R}$ \\
\hline$(60,11,12)_{L R}$ & $(59.973,12.457,9.485)_{L R}$ & $(59.387,13.753,10.754)_{L R}$ & $(58.658,11.549,10.187)_{L R}$ \\
\hline$(59,10,9)_{L R}$ & $(60.580,12.472,9.485)_{L R}$ & $(60.222,13.903,10.754)_{L R}$ & $(59.120,11.719,10.187)_{L R}$ \\
\hline$(54,11,8)_{L R}$ & $(54.633,12.434,9.485)_{L R}$ & $(53.750,13.528,10.754)_{L R}$ & $(53.140,11.549,10.187)_{L R}$ \\
\hline$(61,14,3)_{L R}$ & $(56.998,8.863,8.283)_{L R}$ & $(56.992,10.919,9.500)_{L R}$ & $(56.019,8.653,9.217)_{L R}$ \\
\hline$(34,10,8)_{L R}$ & $(34.060,8.741,8.283)_{L R}$ & $(32.435,9.720,9.500)_{L R}$ & $(32.449,8.449,9.217)_{L R}$ \\
\hline$(38,9,9)_{L R}$ & $(38.818,8.772,8.283)_{L R}$ & $(37.613,10.020,9.500)_{L R}$ & $(37.233,8.585,9.217)_{L R}$ \\
\hline$(64,16,9)_{L R}$ & $(64.026,11.115,9.292)_{L R}$ & $(65.113,13.269,9.608)_{L R}$ & $(65.107,11.068,9.228)_{L R}$ \\
\hline$(56,13,7)_{L R}$ & $(52.726,11.043,9.292)_{L R}$ & $(52.817,12.557,9.608)_{L R}$ & $(53.745,10.745,9.228)_{L R}$ \\
\hline$(63,11,9)_{L R}$ & $(61.647,11.100,9.292)_{L R}$ & $(62.525,13.119,9.608)_{L R}$ & $(62.715,11.000,9.228)_{L R}$ \\
\hline$(66,16,5)_{L R}$ & $(59.633,10.203,9.923)_{L R}$ & $(60.608,11.934,10.492)_{L R}$ & $(59.518,10.865,10.202)_{L R}$ \\
\hline$(49,12,9)_{L R}$ & $(47.738,10.127,9.923)_{L R}$ & $(47.664,11.184,10.492)_{L R}$ & $(47.558,10.526,10.202)_{L R}$ \\
\hline$(55,10,12)_{L R}$ & $(56.064,10.180,9.923)_{L R}$ & $(56.725,11.709,10.492)_{L R}$ & $(55.930,10.763,10.202)_{L R}$ \\
\hline$(67,11,14)_{L R}$ & $(68.175,12.176,6.479)_{L R}$ & $(67.391,12.811,9.068)_{L R}$ & $(66.661,10.661,8.591)_{L R}$ \\
\hline$(53,10,9)_{L R}$ & $(55.091,12.092,6.479)_{L R}$ & $(53.152,11.986,9.068)_{L R}$ & $(53.505,10.287,8.591)_{L R}$ \\
\hline$(54,9,10)_{L R}$ & $(56.877,12.104,6.479)_{L R}$ & $(55.094,12.099,9.068)_{L R}$ & $(55.299,10.338,8.591)_{L R}$ \\
\hline$(70,13,7)_{L R}$ & $(68.807,10.989,8.026)_{L R}$ & $(68.181,13.111,8.901)_{L R}$ & $(68.952,11.114,8.459)_{L R}$ \\
\hline$(68,9,10)_{L R}$ & $(69.995,10.996,8.026)_{L R}$ & $(69.475,13.186,8.901)_{L R}$ & $(70.148,11.148,8.459)_{L R}$ \\
\hline$(65,10,9)_{L R}$ & $(65.834,10.970,8.026)_{L R}$ & $(64.945,12.923,8.901)_{L R}$ & $(65.962,11.029,8.459)_{L R}$ \\
\hline$(75,5,14)_{L R}$ & $(82.242,9.475,7.819)_{L R}$ & $(81.020,11.020,8.438)_{L R}$ & $(82.602,9.435,7.863)_{L R}$ \\
\hline$(84,10,7)_{L R}$ & $(82.837,9.479,7.819)_{L R}$ & $(81.667,11.057,8.438)_{L R}$ & $(83.200,9.452,7.863)_{L R}$ \\
\hline$(80,12,6)_{L R}$ & $(77.484,9.445,7.819)_{L R}$ & $(75.842,10.720,8.438)_{L R}$ & $(77.818,9.299,7.863)_{L R}$ \\
\hline Overall distance & 113.448 & 142.3720 & 109.9628 \\
\hline
\end{tabular}

$$
d_{T}=\sum_{i=1}^{m} d\left(\tilde{y}_{i}^{o b s}, \tilde{y}_{i}^{\text {pred }}\right)
$$

In order to compare the predicted values, first Li et al.'s [18] solution is converted to LR representation of fuzzy numbers, since the authors used a different representation. From the last row of Table 2, it can be seen that the model obtained by the Linear Scalarisation Method (FFLP problem (l-P1)) has the smallest overall distance value, followed by Li et al.'s [18] model and the model obtained by using the proposed Chebyshev Scalarisation Method. 


\section{Concluding Remarks}

In this paper, we proposed two methods for FFLR analysis. Contrary to existing methodologies that use crisp-valued distance functions, we formulated the FFLR problem as a FFMOLP problem. Fuzzy linear and Chebyshev scalarisations were proposed to solve the FFMOLP problem using a lexicographic method for FFLP.

The proposed methods were compared with a recently published method and showed promising results. In a future work, we plan to conduct an extensive simulation study and consider real-world applications to gain more insights into the performance of the proposed methods. In addition, the use of fuzzy-valued distance functions for FFLR analysis will be investigated.

Acknowledgements. The research of José Luis Verdegay is supported in part by project TIN2017-86647-P (Spanish Ministry of Economy and Competitiveness and FEDER funds from the European Union).

\section{References}

1. Azadeh, A., Khakestani, M., Saberi, M.: A flexible fuzzy regression algorithm for forecasting oil consumption estimation. Energy Policy 37(12) (2009). https://doi. org/10.1016/j.enpol.2009.08.017

2. Chang, P.T., Lee, E.S.: Fuzzy least absolute deviations regression based on the ranking of fuzzy numbers. In: Proceedings of 1994 IEEE 3rd International Fuzzy Systems Conference, pp. 1365-1369. IEEE (1994). https://doi.org/10.1109/ FUZZY.1994.343613

3. Chang, P.T., Lee, E.S.: Fuzzy linear regression with spreads unrestricted in sign. Comput. Math. Appl. 28(4), 61-70 (1994). https://doi.org/10.1016/08981221(94)00127-8

4. Chen, L.h., Hsueh, C.C.: Fuzzy regression models using the least-squares method based on the concept of distance. IEEE Trans. Fuzzy Syst. 17(6), 1259-1272 (2009). https://doi.org/10.1109/TFUZZ.2009.2026891

5. Chen, T., Wang, M.J.J.: Forecasting methods using fuzzy concepts. Fuzzy Sets Syst. 105(3), 339-352 (1999). https://doi.org/10.1016/S0165-0114(97)00265-0

6. Chukhrova, N., Johannssen, A.: Fuzzy regression analysis: systematic review and bibliography. Appl. Soft Comput. J. 84, 105708 (2019). https://doi.org/10.1016/ j.asoc.2019.105708

7. Das, S.K., Mandal, T., Edalatpanah, S.A.: A mathematical model for solving fully fuzzy linear programming problem with trapezoidal fuzzy numbers. Appl. Intell. 1-11 (2016). https://doi.org/10.1007/s10489-016-0779-x

8. Dubois, D., Prade, H.: Operations on fuzzy numbers. Int. J. Syst. Sci. 9(6), 613-626 (1978). https://doi.org/10.1080/00207727808941724

9. Ebrahimnejad, A.: An effective computational attempt for solving fully fuzzy linear programming using MOLP problem. J. Ind. Prod. Eng. 36(2), 59-69 (2019). https://doi.org/10.1080/21681015.2019.1585391

10. Ezzati, R., Khorram, E., Enayati, R.: A new algorithm to solve fully fuzzy linear programming problems using the MOLP problem. Appl. Math. Model. 39(12), 3183-3193 (2015). https://doi.org/10.1016/j.apm.2013.03.014 
11. Farhadinia, B.: Ranking fuzzy numbers based on lexicographical ordering. Int. J. Appl. Math. Comput. Sci. 5(4), 248-251 (2009)

12. Hashemi, S.M., Modarres, M., Nasrabadi, E., Nasrabadi, M.M.: Fully fuzzified linear programming, solution and duality. J. Intell. Fuzzy Syst. 17(1), 253-261 (2006)

13. Hosseinzadeh Lotfi, F., Allahviranloo, T., Alimardani Jondabeh, M., Alizadeh, L.: Solving a full fuzzy linear programming using lexicography method and fuzzy approximate solution. Appl. Math. Model. 33, 3151-3156 (2009). https://doi.org/ 10.1016/j.apm.2008.10.020

14. İçen, D., Cattaneo, M.E.G.V.: Different distance measures for fuzzy linear regression with Monte Carlo methods. Soft Comput. 21(22), 6687-6697 (2016). https:// doi.org/10.1007/s00500-016-2218-7

15. Kaur, J., Kumar, A.: Mehar's method for solving fully fuzzy linear programming problems with L-R fuzzy parameters. Appl. Math. Model. 37(12-13), 7142-7153 (2013). https://doi.org/10.1016/j.apm.2013.01.040

16. Kaur, J., Kumar, A.: A new method to find the unique fuzzy optimal value of fuzzy linear programming problems. J. Optim. Theory Appl. 156, 529-534 (2013). https://doi.org/10.1007/s10957-012-0132-4

17. Kelkinnama, M., Taheri, S.M.: Fuzzy least-absolutes regression using shape preserving operations. Inf. Sci. 214, 105-120 (2012). https://doi.org/10.1016/j.ins. 2012.04.017

18. Li, J., Zeng, W., Xie, J., Yin, Q.: A new fuzzy regression model based on least absolute deviation. Eng. Appl. Artif. Intell. 52, 54-64 (2016). https://doi.org/10. 1016/j.engappai.2016.02.009

19. Pérez-Cañedo, B., Concepción-Morales, E.R.: A method to find the unique optimal fuzzy value of fully fuzzy linear programming problems with inequality constraints having unrestricted L-R fuzzy parameters and decision variables. Expert Syst. Appl. 123, 256-269 (2019). https://doi.org/10.1016/j.eswa.2019.01.041

20. Pérez-Cañedo, B., Verdegay, J.L., Miranda Pérez, R.: An epsilon-constraint method for fully fuzzy multiobjective linear programming. Int. J. Intell. Syst. 35(4), 600-624 (2020). https://doi.org/10.1002/int.22219

21. Profillidis, V.: Econometric and fuzzy models for the forecast of demand in the airport of Rhodes. J. Air Transp. Manag. 6(2), 95-100 (2000). https://doi.org/10. 1016/S0969-6997(99)00026-5

22. Rabiei, M.R., Arghami, N.R., Taheri, S.M., Gildeh, B.S.: Least-squares approach to regression modeling in full interval-valued fuzzy environment. Soft Comput. 18(10), 2043-2059 (2013). https://doi.org/10.1007/s00500-013-1185-5

23. Sakawa, M., Yano, H.: Multiobjective fuzzy linear regression analysis and its application. Electron. Commun. Jpn. (Part III: Fundam. Electron. Sci.) 73(12), 1-10 (1990). https://doi.org/10.1002/ecjc.4430731201

24. Song, K.B., Baek, Y.S., Hong, D., Jang, G.: Short-term load forecasting for the holidays using fuzzy linear regression method. IEEE Trans. Power Syst. 20(1), 96-101 (2005). https://doi.org/10.1109/TPWRS.2004.835632

25. Tanaka, H., Uejima, S., Asai, K.: Linear regression analysis with fuzzy model. IEEE Trans. Syst. Man Cybern. 12(6), 903-907 (1982). https://doi.org/10.1109/TSMC. 1982.4308925

26. Voxman, W.: Some remarks on distances between fuzzy numbers. Fuzzy Sets Syst. 100(1-3), 353-365 (1998). https://doi.org/10.1016/S0165-0114(97)00090-0

27. Wang, M.L., Wang, H.F., Chih-Lung, L.: Ranking fuzzy number based on lexicographic screening procedure. Int. J. Inf. Technol. Decis. Making 4(4), 663-678 (2005). https://doi.org/10.1142/S0219622005001696 
28. Wang, W., Wang, Z.: Total orderings defined on the set of all fuzzy numbers. Fuzzy Sets Syst. 243, 131-141 (2014). https://doi.org/10.1016/j.fss.2013.09.005

29. Wang, X., Kerre, E.E.: Reasonable properties for the ordering of fuzzy quantities (I). Fuzzy Sets Syst. 118(3), 375-385 (2001). https://doi.org/10.1016/S01650114(99)00062-7

30. Wang, X., Kerre, E.E.: Reasonable properties for the ordering of fuzzy quantities (II). Fuzzy Sets Syst. 118(3), 387-405 (2001). https://doi.org/10.1016/S01650114(99)00063-9

31. Zadeh, L.: Fuzzy sets. Inf. Control 8(3), 338-353 (1965). https://doi.org/10.1016/ S0019-9958(65)90241-X

32. Zhou, J., Zhang, H., Gu, Y., Pantelous, A.A.: Affordable levels of house prices using fuzzy linear regression analysis: the case of Shanghai. Soft Comput. 22(16), 5407-5418 (2018). https://doi.org/10.1007/s00500-018-3090-4 